\title{
Modelling and Self-organizing in Mobile Wireless Sensor Networks: Application to Fire Detection
}

\author{
Elie Tagne Fute \\ Systems and Transportation Laboratory \\ University of Technology of Belfort-Montbliard \\ University of Dschang \\ Yaounde, 20269, Cameroon \\ Belfort, 90010, France
}

\author{
Emmanuel Tonye \\ Electronic and Signal Treatment Laboratory \\ University of Yaounde I \\ Yaounde, 8390, Cameroon
}

\begin{abstract}
This paper describes the design of a system for wildfire monitoring incorporating wireless sensors. A wireless sensor network (WSN) is a complex system. In complex systems, multiple aspects interact and influence each other. A vast number of entities are present in the system. Traditional modelling and simulation techniques fail to capture interactions between loosely coupled aspects of a complex system. This paper also describes a modelling approach of the mobile WSN based on Holonic MultiAgent paradigm (Holonic Multi-Agent Systems (HMAS)). The holonic model has the characteristics of scalability and robustness. The approach of modelling used is organizational and it uses four basic concepts: Role, Interaction, Organization and Capacity. This paper mainly presents the formulation problem of fire detection. An application is made to the patrolling task and the detection and avoidance of the obstacles during moving of the mobile sensor.
\end{abstract}

\section{General Terms:}

Sensor, multi-agent, organization, role, interaction, capacity, fire, forest fire, bush fire

\section{Keywords:}

Wireless sensor network, mobile sensors, patrolling, agent, multi-agent system, holon, fire detection

\section{INTRODUCTION}

Wireless sensor networks have been attracting many research efforts during the past few years. Sensor networks, usually composed of a few sinks and a quantity of inexpensive and small sensor nodes, have been deployed in a variety of applications such as habitat monitoring, forest fire detection, etc. Satellite-based monitoring is a popular method to detect forest fire now [10, 14]. But the long scan period and low resolution of satellites restrict the effectiveness of the satellite-based forest fire detection [17]. Moreover, satellites usually cannot forecast forest fires before the fire is spread uncontrollably. In this paper, we explore the use of wireless sensor network technology in real-time forest fire detection. We describe the design of a system for wildfire monitoring incorporating wireless sensors. Fire behavior can change rapidly based on a variety of environmental conditions such as temperature, relative humidity, and wind. Moreover, these environmental conditions can differ signifiantly between topographical features such as elevation and aspect. Hence, the ability to accurately monitor these environmental conditions over a wide area becomes of paramount importance. Our goal is to deploy the sensors, with the aim of detecting the fire instead. This study is composed of sensors regarded as autonomous entities to collaborate in order to ensure their goal. Not having an idea of where the fire will trigger, we employ a strategy patrol sensor. This presence of obstacles in the environment requires the use of an appropriate model for avoiding obstacles. For this, we propose a force-based model. The MAS (Multi-Agents System) approach lends itself well to these types of problems because it has a set of autonomous components that manage their own resources and then to work together to achieve their goal. Our approach is organizational, mostly based on concepts or notions of roles and interactions between roles. The remainder of this paper is organized as follows. Section 2 describes the related work. Section 3 describes the problem description, section 4 presents the fire and network models for forest fire detecting by an organizational approach, section 5 presents the self-deployment by force control and the simulation results. A part of simulation results will be shown in section 6 . Finally, section 7 concludes this paper.

\section{RELATED WORK}

There do not exist many works relative for the event's detection using mobile wireless sensor networks. We got intersested in the state of art of other technics to detect events. Most existing forest-fire detection systems rely on the satellite imagery. MODIS provides global daily forest fire products based on the satellite imagery [14]. Here, events are detected by using the image analysis. We explore the use of wireless sensor network technology in real-time forest fire detection. In [3], the system is composed of environmental sensors collecting temperature, relative humidity and barometric pressure with an on-board GPS unit attached to a wireless network mote. The motes communicate with a base station, which communicates the collected data to software running on a database server. In this case, authors use the fixed sensors to collect data. [4, 12] designed and implemented a Mobile Agent in a Wireless Sensor Network. The itineraries are planned on a powerful computer by a genetic algorithm. In addition, authors suited their solution to generate Agilla mobile agents. [1] developed a forest-fires surveillance system in South Korea Mountains. The Forest-Fires Surveillance System (FFSS) developed consists of WSNs, middleware and web application. The middleware program and the web application analyze the collected data and information. The FFSS is able to detect the heat. It let to know early alarm in real time when the forest-fire occurs.

\section{PROBLEM DESCRIPTION}

Mobile wireless sensors are here used to ensure a dynamic coverage of forest areas in order to detect and control wildfires.

\subsection{Environment Description}

Figure 1 is a typical example of an environment where a network of mobile sensors will patrol. Mobile sensors have to coordinate their actions in order to avoid that this forest area becomes subjected to some wildfires. Recovery places are assumed to be lo- 


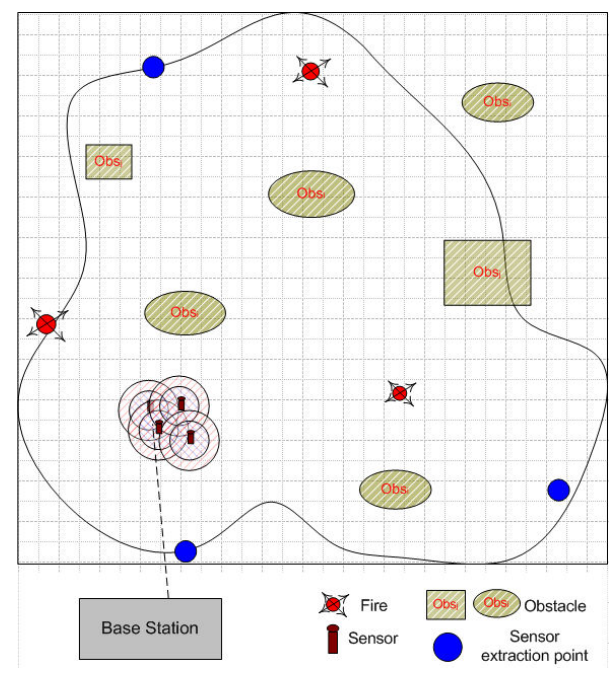

Fig. 1. A typical environment to patrol

cated all around the environment. Sensors that reach their critical energy capacity have to reach a recovery place, so that they can be picked up and then reused after their battery have been filled up again.

Coordination can be ensured by one or several sink nodes. Sink nodes are heavily equipped sensors that gather information from other sensors and communicate this information to a base station. The base station merges all the information received and can send new orders to the sink. The sink node then can communicate these orders to the appropriate sensors.

Detecting wildfires in a forest consists in performing a patrolling task. Patrolling using a mobile wireless sensor network can be divided into two stages. First, sensor nodes are deployed in the environment at a given location. Secondly, they elaborate a formation around a sink node and then start to patrol in the search of fire areas, by maintaining the formation. Indeed, a formation ensures that all the mobile sensors will be able to communicate to each other if it is necessary. Once a fire source is detected by a sensor node, it sends a warning to its sink node, that transmits it to the base station that can finally warn the firefighters. To better help the firefighters, the patrolling sensors can reoganize themselves to follow the fire expansion.

\subsection{Sensor Model}

A mobile sensor node is made up of several components: a sensing unit, a processing unit, a transceiver unit, a power unit, a location finding system and a mobilizer. Sensing units are usually composed of sensors (e.g. thermal, visual, infrared, radio...) and analog to digital converters (ADCs). The analog signals perceived by the sensors are converted to digital signals by the ADCs. Digital signals are then used by the processing unit to make the sensor node collaborate with the other sensor nodes to fulfill the assigned tasks. The transceiver unit (e.g. a radio frequency device) enables a sensor to communicate with other nodes in the network. The power unit supplies current to the other sensor components. It is the most important unit since the lifetime of a sensor network depends on the lifetime of the power resources of each sensor node. Most of the sensor network perception tasks and routing techniques require the knowledge of location with high accuracy, provided by the location finding system. Finally, mobile sensors can move in their environment using a mobilizer.

The position of each moving sensor node is dependent on its interactions with the other sensor nodes and obstacles. Each sensor node is characterized by an occupation radius $r_{o c c}$ that represents the surface it takes on the ground and it can perceive the obsta-

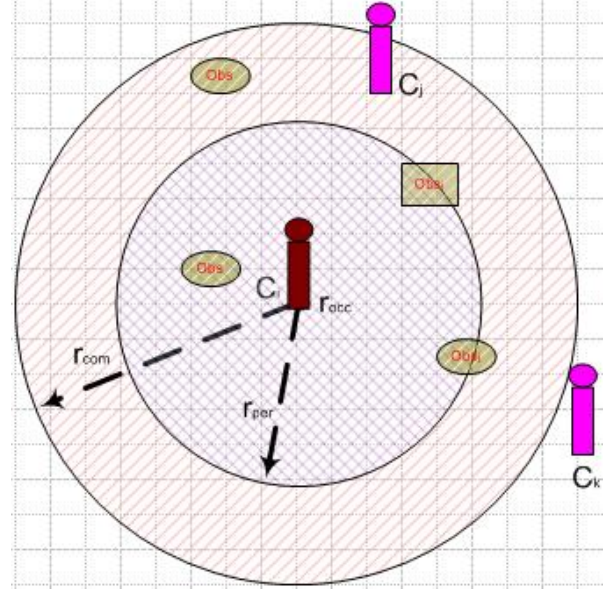

Fig. 2. A sensor model

cles within its perception radius $r_{p e r}$ and the other nodes within its communincation radius $r_{\text {com }}$ (figure 2).

In the next two subsections, the mathematical frameworks of the patrolling task and the energy conservation task are described.

\subsection{Mathematical framework of the patrolling task}

The patrolling problem is usually specified formally as in [11, 2, 9, 8]. Let the graph $G=(V, E)$ where $V$ is the set of nodes and $E$ the edges. The domaine to patrol is generally defined by the graph $G$. Let's consider $p$ sensor agents to visit the nodes of the graph at regular intervals. At the initial time, each of the $p$ agents are located on one node of $V$.

Solving the patrolling problem consists in elaborating a multiagent graph coverage strategy $\pi$. Such a strategy must optimize one or several given quality criteria [9].

$\pi=\left\{\pi_{1} \cdots \pi_{p}\right\}$ is made up of the $p$ individual strategies $\pi_{i}$ of each agent $i$. Let's define an individual strategy by:

$$
\pi_{i}:\left\{\begin{array}{l}
N \rightarrow V \\
\pi_{i}(j+1)=x \text { with }\left(\pi_{i}(j), x\right) \in E \\
\pi_{i}(j) \text { denoting the } j \text {-th node visited by the agent } i
\end{array}\right.
$$

It is commonly admitted that a relevant patrolling strategy is one that minimizes, for each node, the time span between two visits to the same node.

Several criteria have been devised in [11, 6, 8] in order to evaluate the quality of a multi-agent patrolling strategy $\pi$ after $T$ time steps (or cycles) of simulation. All of them are based on the notion of Instantaneous Node Idleness(INI).

The INI $I_{i, t}(\pi)$ of a node $i$ at time $t$ following the multi-agent patrolling strategy $\pi$ is the number of time steps this node remained unvisited. By convention, at the initial instant, $I_{i, 0}(\pi)=0$, $\forall i=1,2, \cdots,|V|$ for any $\pi$.

At a given instant $t, G I_{t}(\pi)$ is the Instantaneous Average Graph Idleness (IGI) when following the multi-agent patrolling strategy $\pi$, i.e.:

$$
G I_{t}(\pi)=\frac{1}{|V|} \sum_{i \in V} I_{i, t}(\pi)
$$

Similarly the Instantaneous Worst Graph Idleness $W I_{t}(\pi)$ is the highest INI encountered since $t$ time steps of simulation when following the multi-agent patrolling strategy $\pi$, i.e.:

$$
W I_{t}(\pi)=\max _{i \in V} I_{i, t}(\pi)
$$

A multi-agent patrolling strategy $\pi$ can be evaluated after $T$ cycles of simulation using either the average idleness criterion 
$A I_{T}(\pi)$ or the worst idleness $W I_{T}(\pi)$. The average idleness denotes the mean of the IGI over the $T$ simulation cycles, i.e.:

$$
A I_{T}(\pi)=\frac{1}{T} \sum_{t=1}^{T} G I_{t}(\pi)
$$

The worst idleness is the highest INI observed during the $T$-time steps of the simulation, given by the following equation:

$$
W I_{T}(\pi)=\max _{t=1,2, \ldots, T} \max _{i \in V} I_{i, t}(\pi)
$$

For both equations, $T$ is the elapsed time until the last sink has reached its critical energy capacity. This critical energy capacity must allow it to perform two important tasks: send its more recent data to the base station and reach the nearest recovery place. Detecting wildfires implies several assumptions. First the environment to patrol will be represented by a regular grid, as the one shown in figure 1 Thus each node of the patrolling graph will be linked by at most eight other nodes. Secondly, ensuring that a fire will not declare itself above a given time limit implies that sensors follow a multi-agent patrolling strategy $\pi^{*}$ that minimizes the worst idleness criteria, i.e.:

$$
\pi^{*}=\operatorname{argmin}_{\pi \in \Pi} W I_{T}(\pi)
$$

where $\Pi$ is the set of the possible multi-agent patrolling strategies.

\subsection{Mathematical framework of the energy conservation task}

Detecting wildfires as long as possible requires that the mobile sensors are able to patrol while minimizing their energy consumption.

The amount of the energy consumed by a sensor $i$ at time $t$ when following a multi-agent patrolling strategy $\pi$ is dependent of several elements and can be defined by the following equation:

$$
E_{i, t}(\pi)=E_{i, t}^{\text {comp }}(\pi)+E_{i, t}^{c o m}(\pi)+E_{i, t}^{\text {perc }}(\pi)+E_{i, t}^{l o c}(\pi)
$$

where:

$-E_{i, t}^{c o m p}(\pi)$ is the energy consumed by the computations carried out between the times $t-1$ and $t$,

$-E_{i, t}^{c o m}(\pi)$ is the energy consumed by the communications performed between the times $t-1$ and $t$,

$-E_{i, t}^{\text {perc }}(\pi)$ is the energy consumed by the perception system between the times $t-1$ and $t$,

$-E_{i, t}^{l o c}(\pi)$ is the energy consumed by the locomotion system between the times $t-1$ and $t$.

All the terms of this equation refer to energy consumptions and they are expressed in Watt/hour.

Hence the minimization of the energy consumed by the network of mobile sensors requires that they follow the best multi-agent patrolling strategy $\pi^{*}$, i.e.:

$$
\pi^{*}=\operatorname{argmin}_{\pi \in \Pi} \max _{t=1,2, \ldots, T} \max _{i=1,2, \ldots, p} E_{i, t}(\pi)
$$

where $\Pi$ is the set of the possible multi-agent patrolling strategies and $T$ is the elapsed time until the last sink has reached its critical energy capacity.

\section{APPLICATION TO FIRE DETECTION}

The objective is to model the problem in terms of organizations, roles, capacities and interactions. CRIO [7] introduces four basic concepts: Role, Interaction, Organization and Capacity. A role is an abstraction of a behavior or a status in an organization. An interaction links two roles in a way that an action in the first role produces a reaction in the second. An organization is defined by a set of roles and their interactions. A capacity is a pure

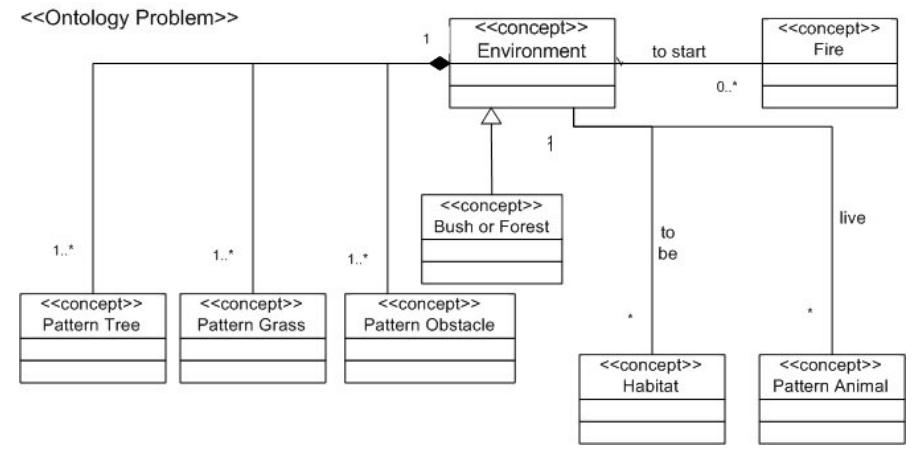

Fig. 3. Ontology model related to the forest/bush fire problem

description of a know-how. A role may require that individuals playing it have some specifics capacities to properly behave as defined. The notion of capacity was introduced to control and exploit these additional behaviors, emerging from roles interactions, by considering an organization as able to provide a capacity. It describes what an organization is able to do [13]. From these concepts an agent is specified as an active communicative entity, which plays roles. An agent may be associated to one or more roles and a role may be played by one or more agents.

\subsection{Fire Problem Modelling}

The main objective is to detect fires and transmit the alert data for rapid intervention. Thus, making the monitoring of forest or bush fire, can give to perform the following sub-objectives:

- Targeting the area to monitor

-Identifying areas of high sensitivity

-Deploying a group of sensors

- Registering data and routing to the central site

-Identifying the fire sources during patrol

- Sending alerts and location data to the central site if detected fire

-Calling intervention teams immediately upon receipt of fire alarms

- Tracking the evolution of fire triggered

-Controlling the surrounding areas to a source of fire

We present an ontology model related to the forest or bush fire problem. In figure 3 , an ontology model related to the fire problem using UML profiles for class diagram is presented.

\subsection{WSN Problem Modelling}

The problem domain is dedicated to the description of a problem independently of a specific solution. We present in figure 4 an ontology model related to the deployment of wireless sensor network.

We present in figure 5 a fragment of solution ontology model. After an ontology model, we must model the problem in terms of organizations, roles, capacities and interactions. Each role is associated with a set of concepts in the ontology, usually a subset of those associated with his organization. By using "Boundary Roles", the scope of application and the border between the system and its environment are refined. We have two large organizations: WSN Simulation and Sink Management. The organization WSN Simulation is responsible for deployment at macro level, and observation network properties. While the organization Sink Management is responsible for achieving the goal specified in the original. Our study will focus on the latter.

It is composed of five roles: Environment, Environment characteristics observer, Strategy, Role Sink, Role Sensor. Figure 6 shows the roles and interactions of each organization. 


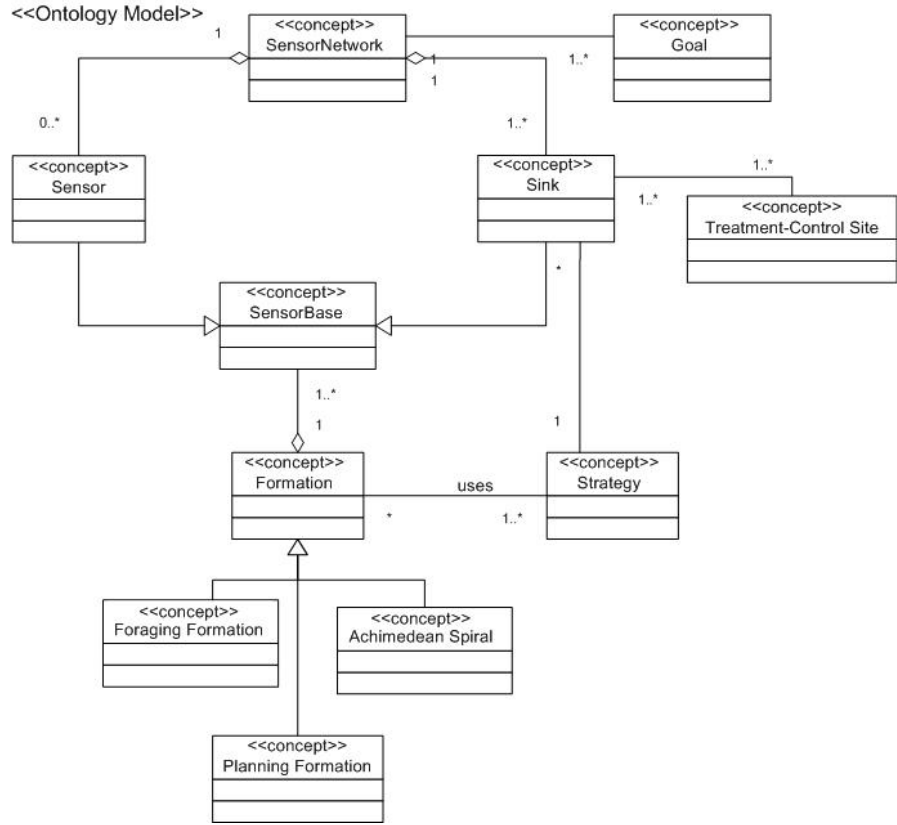

Fig. 4. Ontology model related to the deployment of sensor network

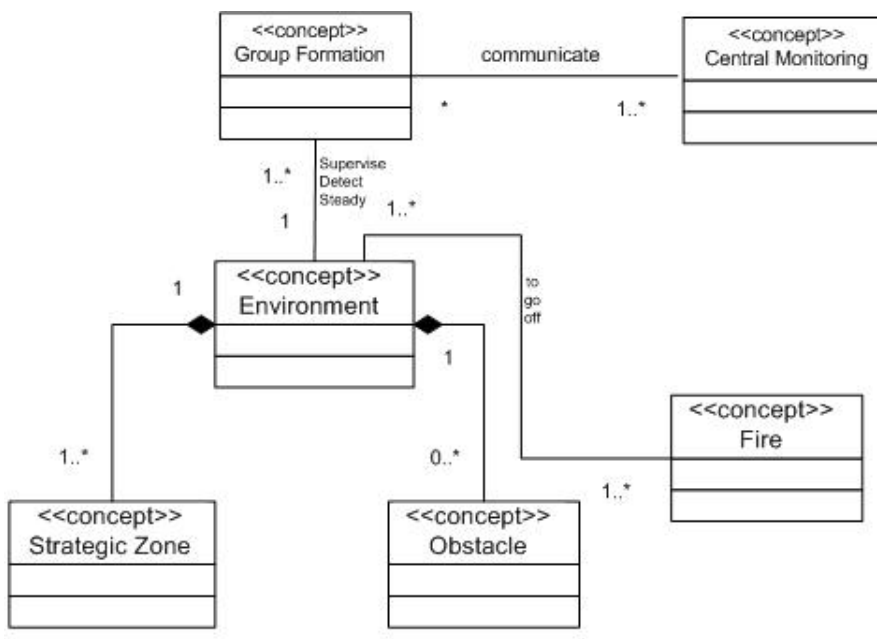

Fig. 5. Solution Ontology model related to the fire detection

\subsection{Agent-Based Solution Design}

After modelling the problem in terms of organizations and roles the objective is, now, to provide a model of the agent society in terms of social interactions and dependencies between entities involved in the solution. Figure 7] shows the communications between roles in Sink Management organization. In organizing Sink Management, we have eight interactions. Three of them, are now modeled by conversations, others are represented by communications. The figures 8 and 9 below show the refinement of the plan behavior of roles Sink and Sensor respectively, these behaviors are transcribed in the form of statechart.

A holon is a self-similar structure composed of holons as substructures. A hierarchical structure composed of holons is called a holarchy [15 5]. The Holonic Organization (HO) represents the organization of a single super-holon at a specific level in the holarchy [16]. The concept of Holon is specialized from the Agent [13]. This definition of holon integrates the production of holonic aspects, the approach being described as organizational. The holonic aspect considers how members organize and manage the super-holon. A specific organization, called Holonic or-

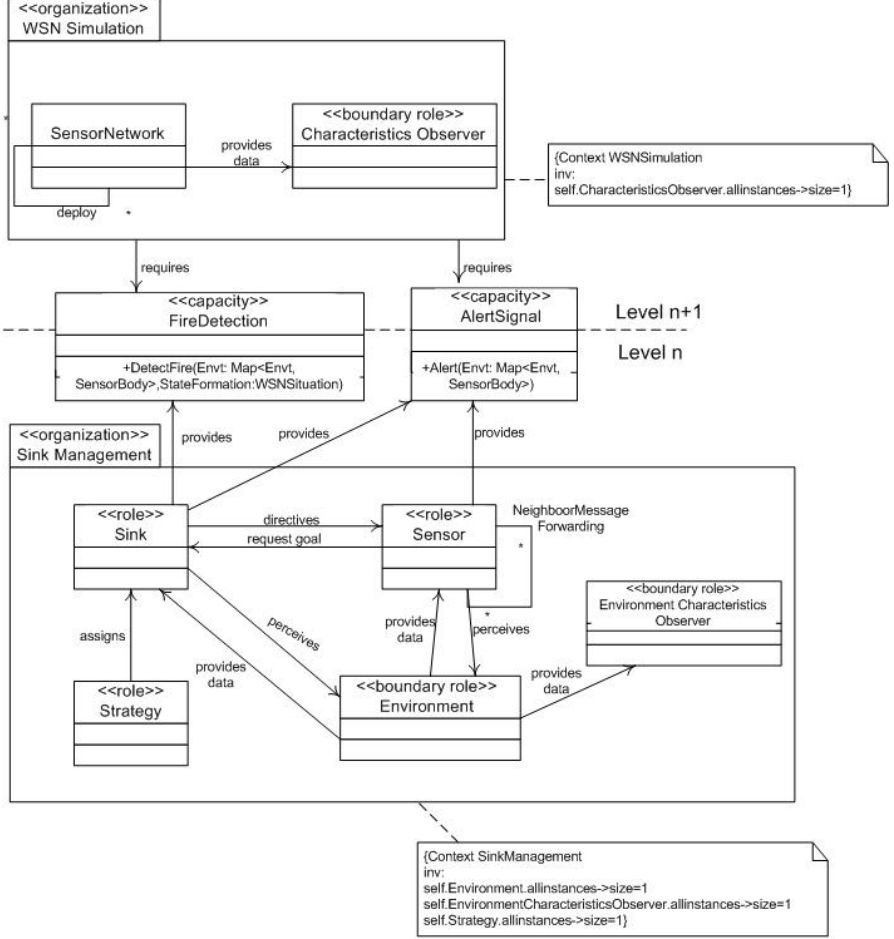

Fig. 6. Organizations Descriptions

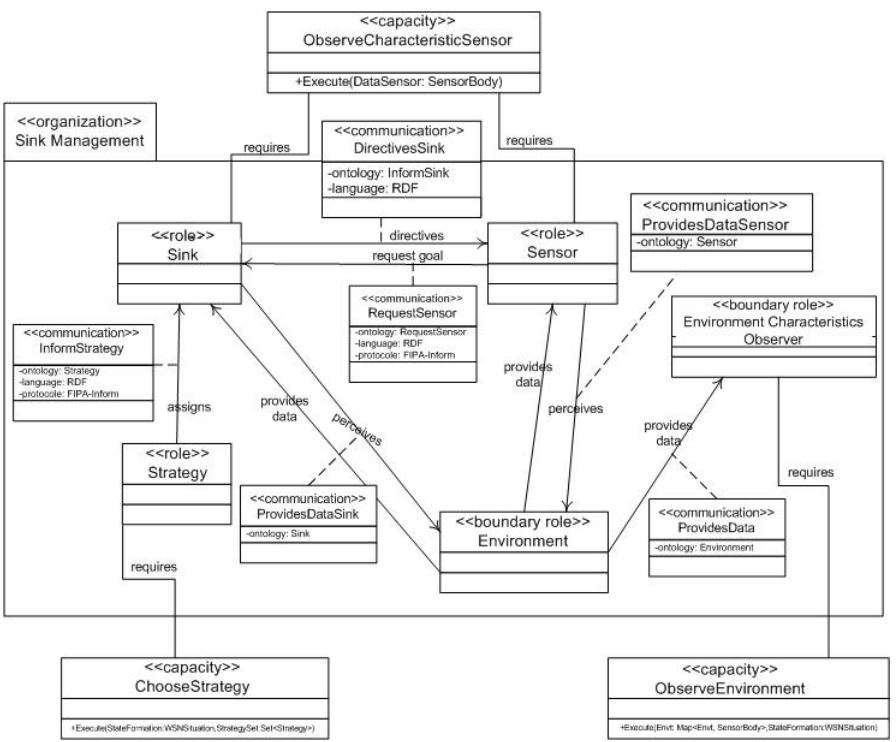

Fig. 7. Communications between roles in Sink Management organization

ganization, is defined to describe the management of a holon and its structure. Depending on the level of abstraction, a super-holon can be considered as an atomic entity (at level $\mathrm{n}$ ) or as an organization of holons (at level n-1) [13]. In multiagent systems, the vision of holons is much closer to the one that MAS researchers have of Recursive or Composed agents. A holon is thus a selfsimilar structure composed of holons as sub-structures and the hierarchical structure composed of holons is called a holarchy. Figure 10 presents our holarchy structure.

\subsection{Implementing Solution Domain}

This part of the model is related to the Holon Implementation Model; its objective is to provide an implementation model of the 


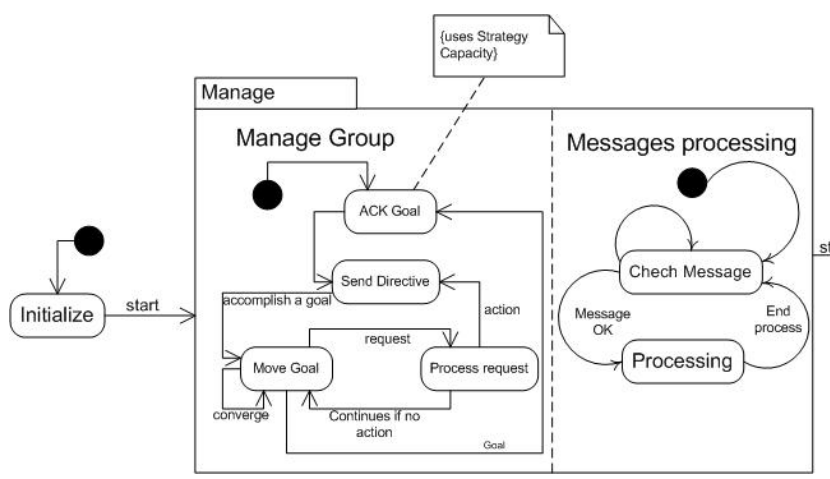

Fig. 8. Statechart of Sink role in Sink Management organization

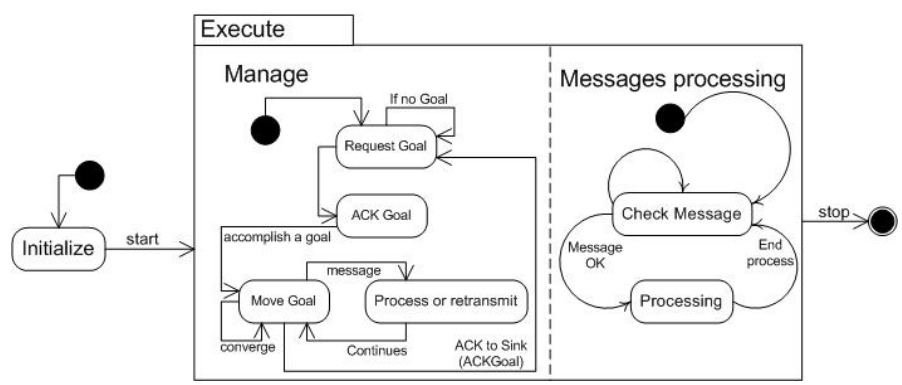

Fig. 9. Statechart of Sensor role in Sink Management organization

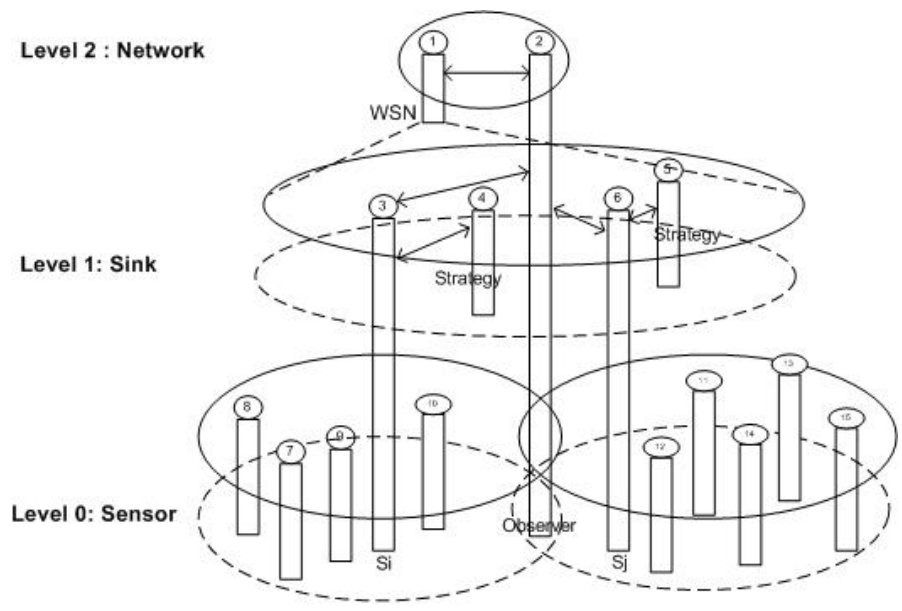

Fig. 10. Holarchy structure

solution. This part is thus dependent on the chosen implementation and deployment platform. Figure 11 presents our model implementing solution. It is an extension of Janus model implementation.

\section{SELF-DEPLOYMENT BY FORCE CONTROL}

Figure 12 shows a sensor agent with a representation of :

-Its communication area (radius $r_{\text {com }}$ ),

-Its area of perception (radius $r_{p e r}$ ),

-Its zone of occupation on the ground (radius $r_{o c c}$ ),

-Obstacles and other sensors located in the areas of communication or perception.

The behavior of a sensor (respectively a group of sensors) is based on three forces:

(1) the attraction force towards the goal, (equation 1),

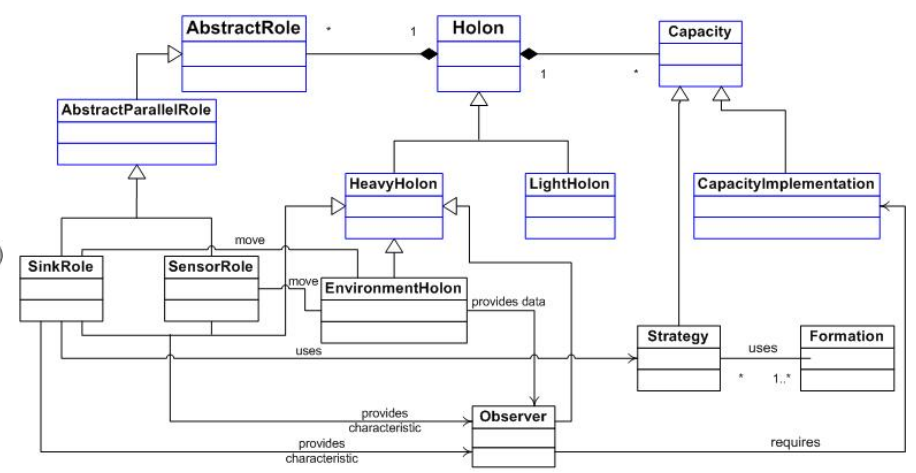

Fig. 11. Solution architecture

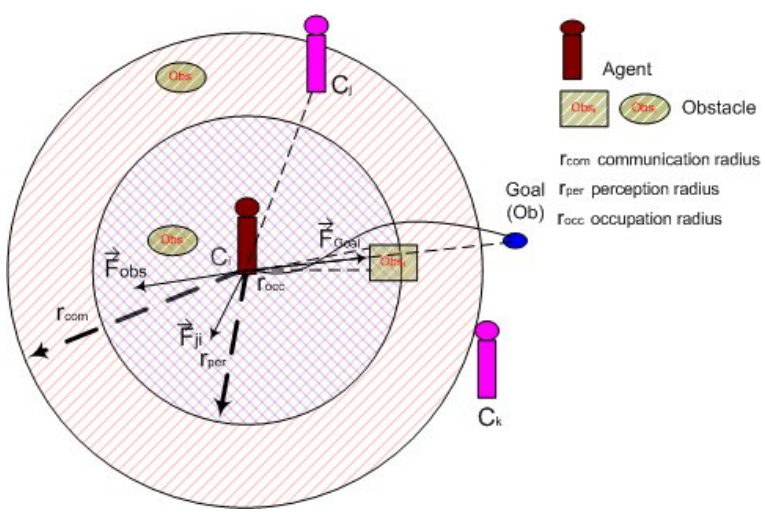

Fig. 12. Forces of a sensor agent

(2) the resultant force of repulsion from other sensors (equation 2 and 5),

(3) the resultant force of repulsion from obstacles of the environment (equation 3 and 4).

$$
\begin{aligned}
& \vec{F}=\delta_{\text {object }} \overrightarrow{C_{i} O \vec{b}}
\end{aligned}
$$

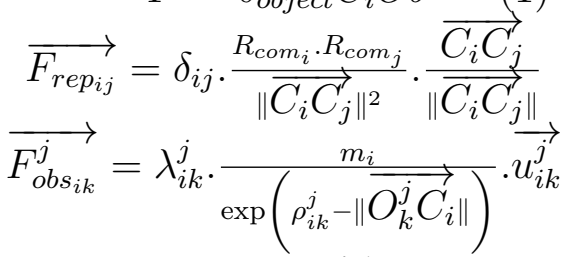

$$
\begin{aligned}
& \text { with } \\
& \overrightarrow{u_{i k}^{j}}=\left(\begin{array}{c}
\cos \left(\theta_{k i}^{j}\right)-\sin \left(\theta_{k i}^{j}\right) \\
\sin \left(\theta_{k i}^{j}\right)
\end{array}\right) \cdot \frac{\overrightarrow{O_{k}^{j} C_{i}}}{\overrightarrow{O_{k}^{j} C_{i} \|}} \\
& \theta_{k i}^{j}=\operatorname{Angle}\left(\overrightarrow{C_{i} O_{k}^{j}}, \overrightarrow{C_{i} O b}\right)
\end{aligned}
$$

Therefore

$$
\begin{aligned}
& \overrightarrow{F_{\text {obs }}}=\sum_{M}^{j=1} \overrightarrow{F_{o b s_{i k}}^{j}} \\
& \overrightarrow{F_{r e p_{i}}}=\sum_{Q}^{j=1} \frac{\overrightarrow{F_{r e p_{i j}}}}{\longrightarrow}
\end{aligned}
$$

$\mathrm{M}$ is the number of points of impact on an obstacle localized in the perception area. Q is the number of agents localized in the communicational area. Since the agent is in motion, we determine the characteristic of his movement by equation 6 :

$$
\begin{gathered}
\overrightarrow{F_{H_{i}}}=\sum_{3}^{k=1} \overrightarrow{F_{i}^{k}}=\overrightarrow{F_{\text {object }}}+\overrightarrow{F_{\text {obs }}}+\overrightarrow{F_{\text {rep }}}= \\
m_{i} \vec{\gamma} \quad(6)
\end{gathered}
$$




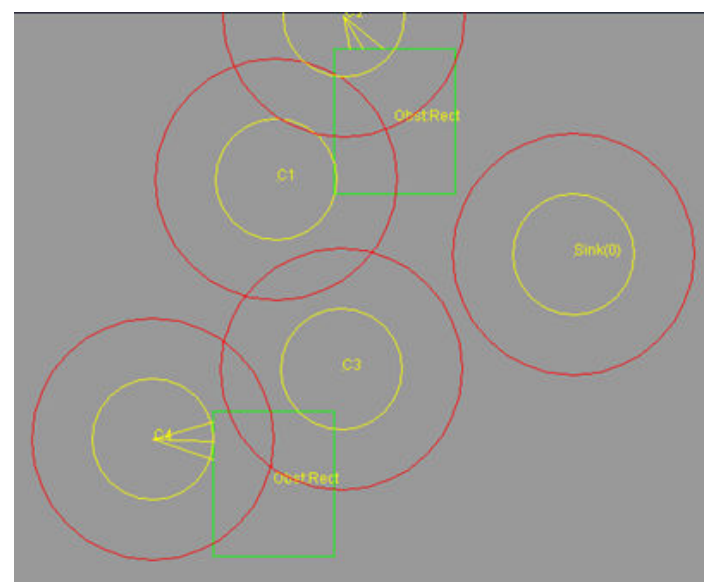

Fig. 13. Motion and detection of the obstacles

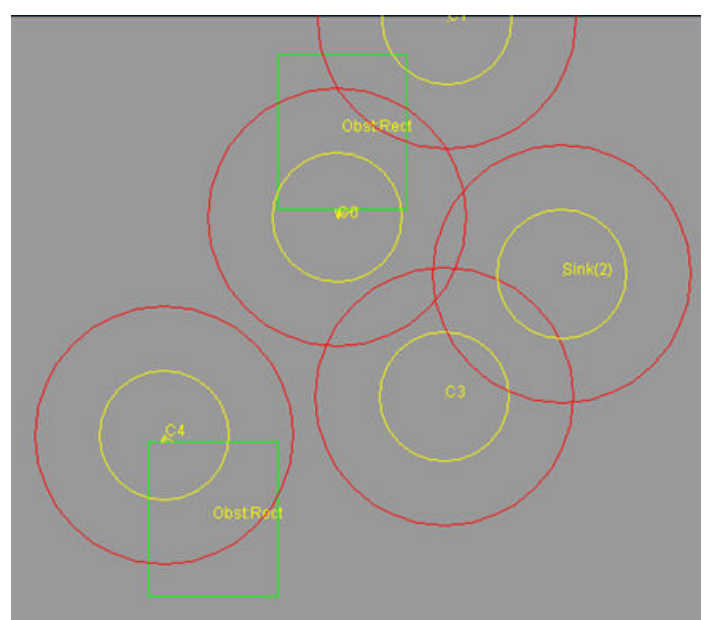

Fig. 14. Motion and avoidance of the obstacles

Instantaneous and Worst Idleness (10 sensors )

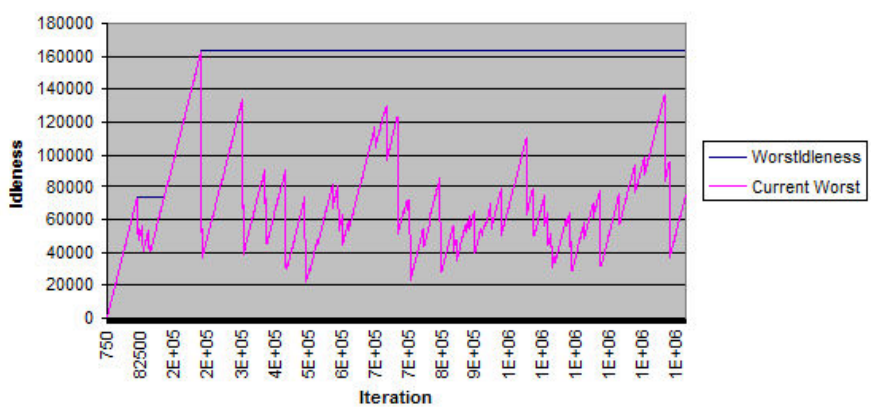

Fig. 15. Worst and instantaneous idleness plots

An application of the force to detect and to avoid the obstacles is presented in figure 13 and 14 respectively. The force model defined allows to properly detect and avoid the obstacles by the sensors during their motion. The simulations show that by using this force model, the mobiles sensors detect and avoid very well the obstacles and other sensors considered like obstacles.

\section{EXPERIMENTAL RESULT}

Figure 15 presents evolution of the worst and instantaneous idleness.
Consommation Energy (10 sensors)

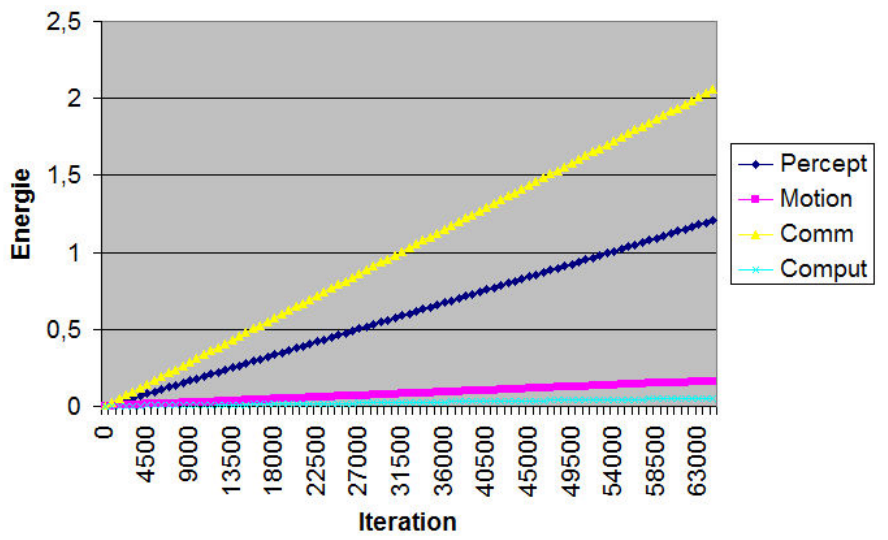

Fig. 16. Energy consumption

Figure 16 presents the plot of the energy consumption. From the curves in figure 16 the communications require more energy than the other tasks of sensors. In terms of energy consumption, we have respectively perception, locomotion and computations. consumption, we have respectively perception, locomotion and computations.

\section{CONCLUSION}

In this paper, we proposed Forest-Fire Detection System based on Wireless Sensor Networks using an organizational approach. Firstly the problem was formulated, then it was modelled using the role concept. An ontology model related to the deployment of mobile wireless sensor networks was also presented. To avoid the obstacles in the environment, a force-based model was used since we had shown that this model detects obstacles with effectiveness and efficiency. The values of Idleness of environment and energy consumption by sensors and Sink were evaluated.

\section{REFERENCES}

[1] S. Byungrak, Yong sork Her, and K. Jung-Gyu. A Design and Implementation of Forest-Fires Surveillance System based on Wireless Sensor Networks for South Korea Mountains. In IJCSNS International Journal of Computer Science and Network Security, Vol.6 No.9B, 2006.

[2] Y. Chevaleyre. Theoretical Analysis of the Multi-Agent Patrolling Problem. In IAT, 2004.

[3] D. M. Doolin and N. Sitar. Wireless sensors for wildfire monitoring. In Proceedings of the SPIE, Vol.5765, pages 477-484, 2005.

[4] Chien-Liang Fok. Efficient Fire Detection and Tracking Using Mobile Agents in a Wireless Sensor Network. In MURI ONR 3-Year Review, Baltimore, 2004.

[5] E. T. Fute, F. Lauri, A. Koukam, and E. Tonye. The Coverage Problem in Wireless Sensor Networks by Holonic Multi-agent Approach. In International Journal of Computing and ICT Research, IJCIR, ACM, ISSN 1818-1139 (Print), ISSN 1996-1065 (Online), Vol.3, No.1, pages 3241, 2009.

[6] E. T. Fute, E. Tonye, A. Koukam, and F. Lauri. Multi-Agent Patrolling Strategy: Application to the Exploration Environment Problem. In International Journal of Automation, Robotics and Autonomous Systems, ICGST-ARAS, ISSN 1687-4811 (Print), 1687-482X (Online), Vol.9, Issue 2, 2009. 
[7] A. Nicolas Gaud. Systmes Multi-Agents Holoniques : De L'analyse L'implantation. PhD thesis, Universit de Technologie de Belfort-Montbliard, 2007.

[8] Arnaud Glad. Etude de l'auto-organisation dans les algorithmes de patrouille multi-agent fondes sur les phromones digitales. PhD thesis, Universit Nancy 2, 2011.

[9] F. Lauri and A. Koukam. A Two-Step Evolutionary and ACO Approach for Solving the Multi-Agent Patrolling Problem. In WCCI, Hong-Kong, China, 2008.

[10] Z. Li, S. Nadon, and J. Cihlar. Satellite detection of Canadian boreal forest fires: development and application of the algorithm. In International Journal of Remote Sensing, Vol.21, no.16, pages 3057-3069, 2000.

[11] A. Machado, G. Ramalho, J. D. Zucker, and A. Drogoul. Multi-Agent Patrolling : an Empirical Analysis of Alternatives Architectures. In Proc. of $3 r d M A B S$, pages 155-170, 2002.

[12] D. Massaguer. Multi Mobile Agent Deployment on Wireless Sensor Networks. In University of California, Irvine,
2005.

[13] C. Massimo, Gaud Nicolas, S. Galland, V. Hilaire, and A. Koukam. A Holonic Metamodel for Agent-Oriented Analysis and Design. In Industrial Applications of Holonic and Multi-Agent Systems: Holonic and Multi-Agent Systems for Manufacturing, pages 237-246, 2007.

[14] MODIS. Moderate-resolution Imaging Spectroradiometer. In MODIS, 2008. http://modis.gsfc.nasa.gov/index.php, visited the 02 july 2008 .

[15] S. Rodriguez, V. Hilaire, and al. A Formal Holonic Framework with Proved Self-Organizing Capabilities. In World Scientific, 2007.

[16] S. Rodriguez, V. Hilaire, and A. Koukam. Toward a Methodological Framework for Holonic multi-agent systems. In ESAW'03, 2003.

[17] Liyang Yu, Neng Wang, and Xiaoqiao Meng. Real-time Forest Fire Detection with Wireless Sensor Networks, IEEE. In proc IEEE MABS, 2005. 